\title{
Models of impulsive phenomena: Experiences with writing an interactive textbook
}

\author{
JÁNOS KARSAI
}

Abstract. "Take the textbook to computer" - is said quite often. Would it be so easy? If we start such a work, we meet a lot of trouble very soon. A book stored on a CD, read on the screen of computer and containing some hyperlinks does not become automatically electronic textbook. There are difficulties also in writing merely an electronic attachment to a classical book. In this paper, we deal with some important features (actually important from our point of view) of interactive mathematics textbooks, arising mathematical, didactical and technical problems. The "principles" are illustrated with examples taken from the book-CD "Models of Impulsive Phenomena".

Key words and phrases: computer-aided learning, hypermedia, interactive textbook, Mathematica.

ZDM Subject Classification: U50, U70.

\section{Introduction}

A cornerstone of computer-aided teaching is to have appropriate electronic handouts and projects for classroom applications. It is also necessary to develop projects for the individual study and research. In [8], we dealt with some possible methods and tools of the computer aid of mathematics education and modelling, and we also considered the requirements for electronic classroom materials. Now we deal with some concerns of the individual work.

There are several possible directions to use computing tools in the learning process. Some nice experiments can be found in [2], [8], [11], [12], [14], [22] and 
the references therein. One of the directions faces towards complete computerization and prefers almost exclusively electronically stored and read "textbooks" to classical ones. We would like to believe that it will not happen for a while. On the other hand, electronic textbooks have a very important role in the education. There have been developed many applications, some of them are absolutely perfect (see the citations), but a lot of them are far from being ideal, especially in the case of mathematics.

In writing and developing the book-CD cited in the title of this paper, we tried to produce a material with unified style and structure that helps to concentrate on the investigated theory while it uses a lot of technical tools. Here, we summarize the concepts followed, and then show examples to illustrate the implementation.

\section{Hypermedia based "static" textbooks}

First of all, we need to point out that it is very tiresome to read a long text on computer screen, although it is necessary to do so very often. Almost everybody (who can do or afford it) makes a printed copy. In spite of this fact, most of the electronic educational materials are of this type because of the following advantages:

- nonprintable animations, sounds, movies and pictures of high resolution can be inserted;

- references are real hyperlinks;

- search functions are available for objects and pieces of the text;

- the cost of publishing is low.

They are very easy to use, since there is no need to use special software and hardly any informatical knowledge from the reader is required. The presence of such kind of multimedia materials more then ten years ago caused a dramatic change in education. The methodology of their development has been standardized by now. In particular, they are very important when the main part of the contained information is audio-visual. Hyperlinks and the search functions help in handling in the textual pieces of information. On the other hand, the reader remains reader, the interaction with the computer concerns and extends only the reading techniques.

Note that the advantages listed above are quite restricted in mathematical materials. Indeed, mathematics books traditionally contain a lot of text and formulas that are not easy to understand. Reading of such texts is much easier 
and less tiresome in printed form than on the computer screen. Now, the question is: digitalize or not the classical textbook in such a way. By our experience we can take the following recommendations:

If the material (system) is planned to be a reference book, manual, dictionary, etc., when the main goal is to find particular short pieces of information rather than a comprehensive study, then the digitalization of an earlier printed version, with extending the index and inserting hyperlinks can be satisfactory. Fortunately, the real multimedia systems go far beyond this minimal goal and most of them are complex informatical systems and do not remind to books.

In the case of book-like materials the necessity of readability and the different goals and requirements can mean a good reason to use both electronic and printed versions. They have to be strictly linked. Every point where a multimedia element can be found in the electronic version has to be marked in the printed version. The particular method of developing an electronic multimedia attachment to existing printed books is common, and can help to reanimate many old books believed to be obsolete.

We need to note that even if our multimedia material is standalone and not an electronic attachment, it does not have to compete with the approved theoretical textbooks. Instead, the new features have to be emphasized. It is certain that mathematics students learn the methods of problem solving from the Pólya's books for a long time. On the other hand, we hope that more and more of them will use computers when applying these methods.

\section{Interactive textbooks}

Using mathematical software packages in developing electronic mathematics textbooks means a real break-through. Here the reader is not a reader any more. The interaction goes beyond the reading techniques; it essentially concerns the mathematical subject. The textbook is not a static, unchangeable something, it is only a starting point. Examples can be modified, formulas, algorithms recalculated and figures replotted. Such systems can be called as interactive textbooks $[3],[7],[9],[10]$.

The simplest and sometimes the only way of the usage of mathematical packages is that the applications developed in them are attached to given points of the static multimedia books considered in the previous section. These applications can be run independently or via hyperlinks. 
Another, technically and didactically much more complicated but possibly more effective solution for the reader-user is that when the mathematical programs, program lines are an organic part of the text. Hence the development of interactive textbook is a complex task including the classical textbook writing, multimedia system development as well as

- development of mathematical program packages, applications,

- fitting and merging the hypermedia system and the mathematical programs.

In addition to the requirements and arguments concerning the multimedia systems and electronic teaching materials [8], the following principles should be taken into account during the development:

- The goal of an interactive textbook is twofold: the theory and also its computer implementation have to be treated, but the book itself has to be applicable for that reader who is interested only in the theory.

- Interactivity makes natural to apply constructive, experimental and visualization methods beside or instead of deductive methods being common in mathematical textbooks.

- On the other hand, it is necessary to deal with the dangers and boundaries of the experimental methods.

- Do not assume deep informatical and programming practice from the user. The material has to contain a minimal set of such knowledge.

- The book has to contain theoretical chapters as well as parts that contain experimental treatment and schemes that help the computer-aided problem solving and the computer implementation of the considered mathematical methods.

- Computer plotting is a little bit different from the classical one. Call the attention to this fact every time when it is needed.

- Exercises, problems have to contain parts for manual as well as computeraided work.

- Choose software that has a "reader" version.

What concerns the program-writing and merging the text and program lines, some important principles are as follows:

- Notations should be identical with the traditional mathematical notations. Differences coming from the programming language have to be used in a consequent way. 
- Program lines are parts of the text; they have to be short, user friendly, well readable and self-explaining.

- Emphasize the commands that the user should, can or must not modify when rerun.

- Technical, programming details cannot disturb the learning process. Hence:

- avoid repeated programs,

- develop and use special commands and functions to hide complicated parts.

- Loading of programs has to be uniform.

In the next section we present some examples from the interactive textbook [7] for the ideas stated above. The reader can decide if it was successful or not.

\section{Examples from the "Models of impulsive phenomena"}

It happens quite often in phenomena of the nature that some effects are performed very quickly or instantaneously. In such cases we speak about impulses. Because of the complicated mathematical formal description, the experimental treatment is especially useful and sometimes more convincing than the rigorous mathematical methods. Watching this fact we did not want to write a classical theoretical textbook. We wanted the reader to get acquainted with the basic concepts of impulsive systems through examples and computer experiments and then with some basic methods of the computer-aided modelling. Hence we tried to develop an electronic textbook for interactive experimental usage completely written in Mathematica.

The structure and the treatment follow this idea. The summaries of theoretical concepts, methods and technical tools are followed by experiments, simulations for interesting systems. Based on the experiments we formulate conjectures, statements for the properties. Some proofs of theorems and experimental results are stated as problems to solve, and we suggest to investigate other systems.

We developed program packages in Mathematica to solve and visualize impulsive systems; hence the book is a collection of programs and modelling schemes that helps the user to perform experiments individually.

Both a full electronic and a printed version are prepared. The printed version is supplied with an electronic attachment that contains the colored version of figures, computer animations, packages developed, and an introduction to Mathematica for the beginner users. 
The interactive version can be read with the help of the MathReader, downloadable from the webpage of Wolfram Research (http://www.wolfram.com).

Now, let us show some examples to illustrate the above statements.

4.1. Table of contents: preliminary chapters

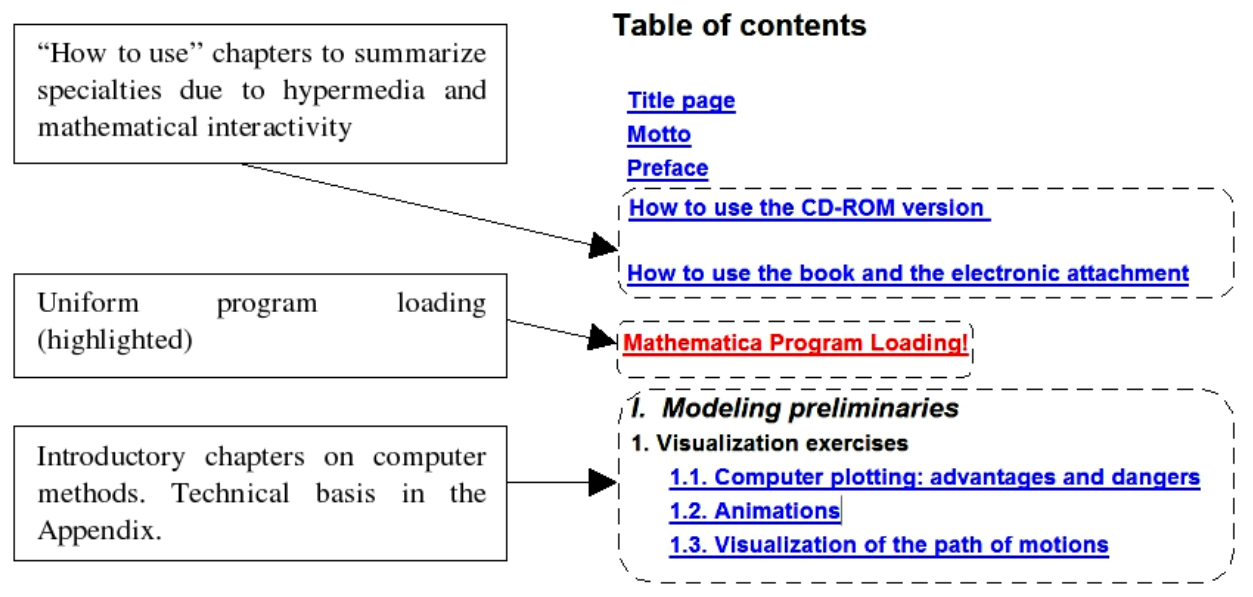

4.2. Table of Contents: treatment of the main parts

\begin{tabular}{|l|l|}
\hline $\begin{array}{l}\text { Theoretical chapters with computer } \\
\text { illustrations }\end{array}$ \\
\begin{tabular}{|l|l} 
Chapters for technical tools and/or \\
methods of computer aided \\
investigation
\end{tabular} \\
$\begin{array}{l}\text { Adaptable modelling schemes for } \\
\text { problem solving }\end{array}$
\end{tabular}


4.3. Table of Contents: a part of the Appendix

\begin{tabular}{|l|}
\hline Program list, loading \\
\hline $\begin{array}{l}\text { Mathematical and special } \\
\text { Mathematica notations. }\end{array}$ \\
\hline Index: list of hyperlinks \\
\hline
\end{tabular}

\subsection{The style and content of chapters}

The general scheme of the structure of chapters is the following:

- detailed treatment or summary of the theory;

- theoretical-experimental study of illustrative examples using Mathematica;

- methods and basic steps of the computer-aided investigation of the given topic shown on examples;

- problems to solve, experiments to perform.

Because of size limitations for this paper we only show here the English translation of a short example that illustrates the phenomenon called "beating".

\section{Beating and passing-through together}

Consider the following simple system:

$$
\begin{gathered}
x^{\prime}=0, \text { if } S_{i}(t, x(\tau-0)) \neq 0, \\
x(\tau+0)=\sqrt{|x(\tau-0)|} \operatorname{sign}(x(\tau-0)), \quad \text { if } S(t, x(\tau-0))=0,
\end{gathered}
$$

where $S(t, x)=\sin (\pi(t-x))$, if $|x| \leq 1$ and let $S(t, x)=1$ otherwise. It is obvious that $\tau_{i}(x)=i+x$, if $|x| \leq 1(i=1,2,3, \ldots)$. It is easy to see that the behavior of solutions starting out of different initial values is different:

- If $\left|x_{0}\right|>1$, then no impulse works on the solution.

- If $\left|x_{0}\right|=1$ or $x_{0}=0$, then the impulse working on the solution is identity.

- If $0<x_{0}<1$, then infinitely many impulses work on the solution at the instans $\tau_{i}$, $\lim _{i \rightarrow \infty} \tau_{i}=1$, and $\lim _{i \rightarrow \infty} x\left(\tau_{i} \pm 0\right)=1$.

- If $-1<x_{0}<0$, then infinitely many impulses work on the solution at the instans $\tau_{i}$, $\lim _{i \rightarrow \infty} \tau_{i}=\infty$, and $\lim _{i \rightarrow \infty} x\left(\tau_{i} \pm 0\right)=1$.

Now, investigate the behavior of the solutions with the help of the program IDERKSolve. 
$\mathbf{x d o t}=\{0\} ; \operatorname{xvar}=\{\mathbf{x}\} ;$

$\mathrm{t} 0=0 ; \mathrm{t} 1=4 ; \mathrm{dt}=0.01 ;$

$\mathbf{x} 1=-2 ; x 2=2$;

init $=\{\{-1\},\{-1.5\},\{-0.1\},\{1\},\{1.5\},\{0.1\}\}$;

$\mathrm{s}\left[t_{-}, x_{-}\right]:=$Which $[\operatorname{Abs}[x]<1, \operatorname{Sin}[\pi(t-x)]$, True, 1$]$;

Impulse $=\{\{\mathrm{S}[t, \mathbf{x}],\{\sqrt{\mathrm{Abs}[\mathrm{x}]} \operatorname{sign}[\mathrm{x}]\}, 1\}\} ;$

Plot the set $S(t, x)=0$ and the mapping by the impulse on it:

Jumpplot $[S[t, x]$, Impulse $[[1,2]],\{t, t 0, t 1\},\{x, x 1, x 2\}$,

plotPoints $\rightarrow 100$, Contourstyle $\rightarrow$ \{Thickness $[0.015]\}]$;

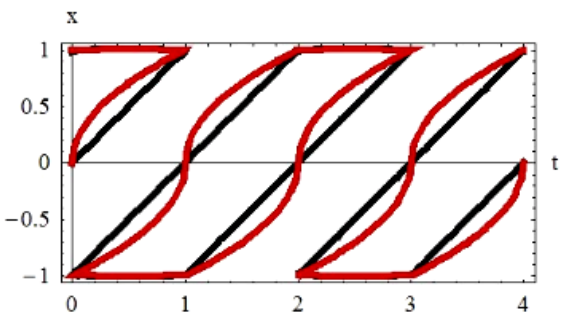

The set $S(t, x)=0$ consists of parallel pieces of straight lines (note that the lines parallel to the $t$-axis appear because of the plotting limitations). First, solve the system:

sol1 = IDERKSolve [xdot, Impulse, xvar, init,$\{t, 0, t 1, d t\}]$;

Plot the solutions and the set $S(t, x)=0$ together:

plt1 $=$ ListPlot1 [ sol1, PlotJoined $\rightarrow$ True,

Gridlines $\rightarrow$ Automatic, PlotStyle $\rightarrow\{$ Thickness $[0.01]\}]$;

plt2 $=$ ContourPlot $[S[t, x],\{t, t 0, t 1\},\{x, x 1, x 2\}$,

Contours $\rightarrow\{0\}$, PlotPoints $\rightarrow 100$, ContourShading $\rightarrow$ False $]$;

Show [plt1, plt2];

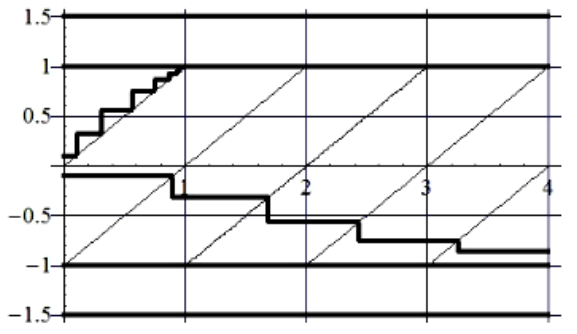

It is visible quite well that beating appears for the values $0<x<1$. The reason is that the impulse enforces solutions to rebound from the surface $S(t, x)=0$. In general, when the coninuabilility of solutions is needed, this phenomenon has to be avoided.

In order to help the readability of the book, we developed special structural and style element. Now, we show them on some parts of a section of the book. To emphasize the structure, we insert a part of the original Hungarian version. 


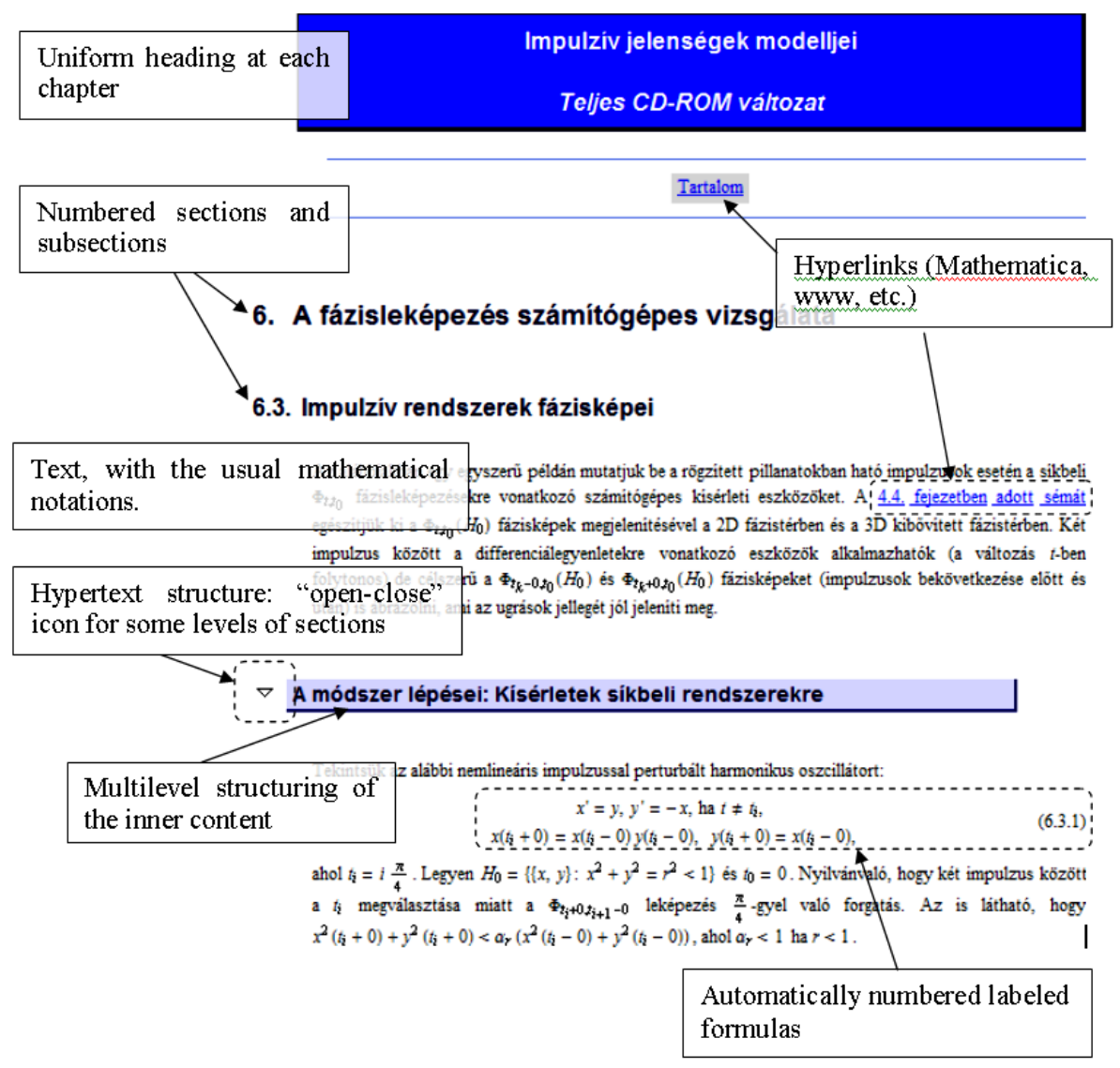

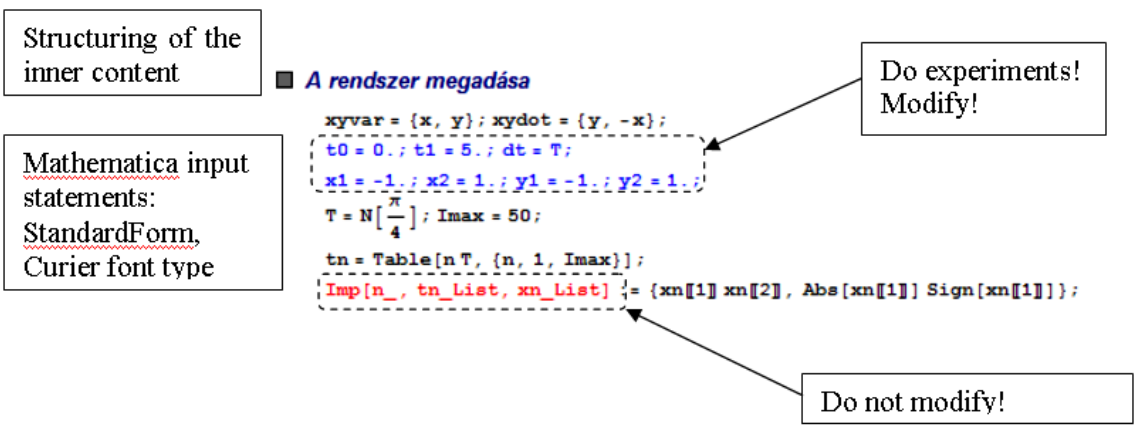


Icon " $E$ ": electronically readable object is at this point in the electronic version and attachment.

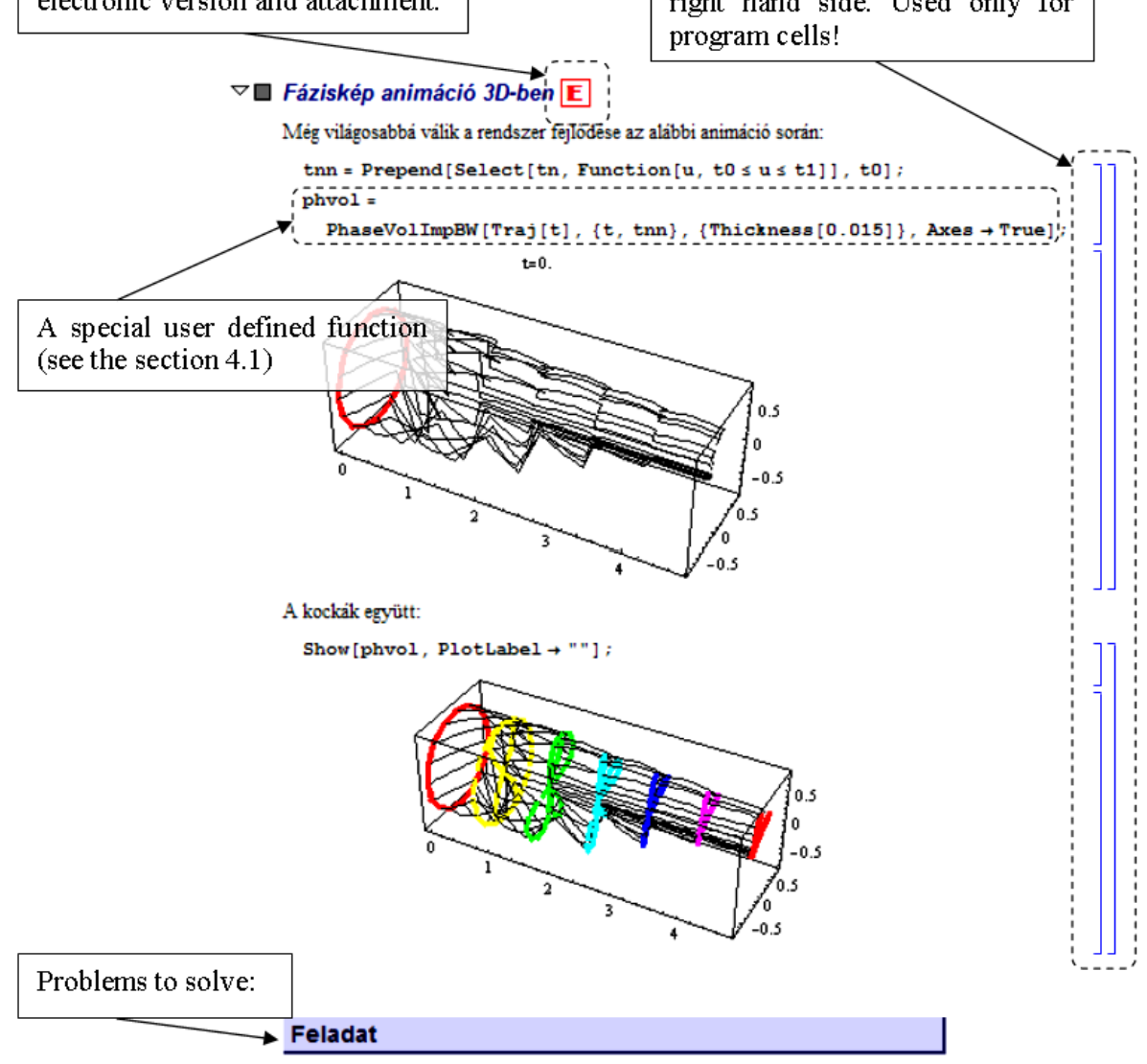

6.3.1. KísÉRLet.

Exercise: solved problem Problem: theoretical problem to solve ok csôkkenésere. Készitsünk animációt, amelyben a kezdeti Experiment: experimental study 


\subsection{Hiding technical details}

Besides packages to investigate impulsive systems, we defined functions for typical operations such as making animations and plots. In such a way, some complicated programs become invisible for the user, so they do not disturb the reading but their use do emphasize the structure of the topic considered.

The function Coordinate extracts two coordinates of a list, which is useful in coordinate-wise plotting.

Coordinate $\left[\right.$ data,$\left.\left\{n_{-}, m_{-}\right\}\right]:=\operatorname{Map}[\{\#[[n]], \#[[m]]\} \&$, data $]$

The function SolPlot plots the coordinates of solutions of a given system stored in the variable Traj.

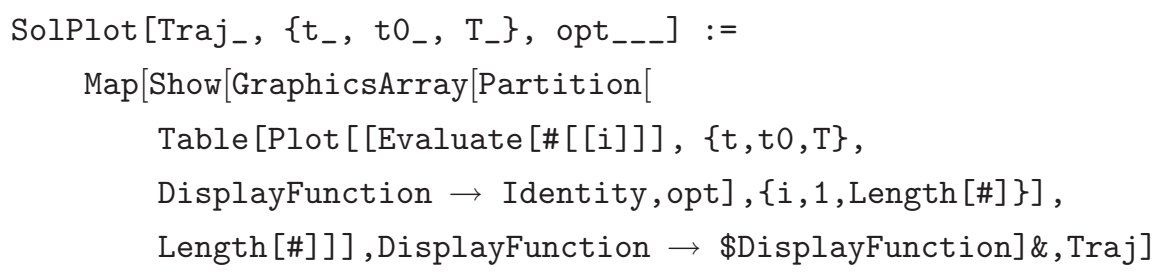

\section{Conclusions}

Based on our experiences with the development and usage of our experimental projects [9] and the book-CDROM [7], we considered some principles of and arising problems at the development of interactive mathematics textbooks.

As summary, we can state that the tasks concerning the development of mathematical programs and inserting them into the mathematical text get a special importance in addition to the classical tasks of writing and multimedia development. Our cited examples give a possible solution for the arisen problems.

ACKNOWLEDGEMENT. The research is supported by Hungarian National Foundation for Scientific Research Grant no. T 034275. 


\section{References}

[1] F. R. Giordano, M. D. Weir and W. P. Fox, A First Course in Mathematical Modeling, Brooks/Cole Publishing Company, 1997.

[2] K. Fuchs and A. Dominik, Mathematica Palettes - Eine für den mathematisch naturwissenschaftlichen Unterricht adaptierte/adaptierbare Computeralgebra Lernumgebung, ÖMG Didaktikhefte, Heft 32, (2000), 24-40.

[3] R. J. Gaylord and P. R. Wellin, Computer Simulations with Mathematica, Telos-Springer, 1995.

[4] A. Gray, M. Mezzino and M. A. Pinsky, Introduction to Ordinary Differential Equations with Mathematica, Springer-TELOS, 1997.

[5] H. C. Hege and K. Polthier (eds.), Visualization and Mathematics, Experiments, Simulations and Environments, Springer, 1997.

[6] J. Karsai, Mathematics for Pharmacy Students, A. Szent-Görgyi Med. Univ., 1996 (in Hungarian).

[7] J. Karsai, Mathematical models of impulsive phenomena, Mathematica experiments and simulations, TypoTEX Budapest, 2002, http://silver.szote.u-szeged.hu/impulse.

[8] J. Karsai, É. V. Rácz, A. Schwenk and N. Kalus, Visualization and Art in the Mathematics Classroom, Zentralblatt für Didaktik der Mathematik 35 (2003), 24-29.

[9] J. Karsai and É. V. Rácz, Computer-aided mathematical modelling with Mathematica, CD-ROM for an intensive course, 2003.

[10] M. M. Neumann and T. L. Miller, Mathematica projects for vector calculus, Kendall/Hunt Publ. Company, 1996.

[11] I. Perjési-Hámori and M. Klincsik, An Interactive Method of Teaching Vectorcalculus via Internet, ODL Networking for Quality Learning, Conference Proceedings, Lisbon 2000, 67-71.

[12] R. Simonovits, Differentialrechnung mit M@th Desktop, ÖMG Didaktikhefte, Heft 33, (2001), 130-139.

[13] S. Wolfram, Mathematica, A System for Doing Mathematics by Computer, Addison-Wesley Publishing Company, 1994.

[14] T. Yoshioka, K. Fuchs, A. Dominik and H. Nisizwawa, Remedial Education of Symbolic Fractional Calculations Focused on Each Calculating Step, In: Proceedings of the Sixth Asian Technology Conference in Mathematics, Melbourne, Australia, 2001, 205-212.

\section{Web sites}

[15] Indiana University of Pennsylvania, Mathematics Department, http://www.ma.iup.edu/projects/.

[16] List of Mathematica related websites, http://smc.vnet.net/mathsite.html.

[17] Maple Application Center, http://www.mapleapps.com/.

[18] Maple Student Center, http://www.maple4students.com/. 
[19] University of Illinois, Urbana - Champaign, Calculus \& Mathematica, http://www-cm.math.uiuc. edu.

[20] University of Pécs, Pollack M. College of Engineering, Dept. Mathematics, http://www.matserv.pmmf.hu/.

[21] University of Szeged, Department of Medical Informatics, http://silver.szote.u-szeged.hu/dmi/karsai/math/.

[22] Wolfram Research Infocenter, http://library.wolfram.com/infocenter/.

JÁNOS KARSAI

DEPARTMENT OF MEDICAL INFORMATICS

UNIVERSITY OF SZEGED

HUNGARY

(Received July, 2003) 